\title{
A descriptive-comparative study of medications used by older people prior to and following admission to a continuing care facility
}

\author{
Alysha Visram ${ }^{1}$, Donna Wilson ${ }^{2}$ \\ ${ }^{1}$ Center for Health Promotion Studies, School of Public Health, University of Alberta, Edmonton, Canada \\ ${ }^{2}$ Faculty of Nursing, University of Alberta, Edmonton, Canada \\ Email: alysha.visram@ualberta.ca, donna.wilson@ualberta.ca
}

Received 10 September 2011; revised 22 October 2011; accepted 10 February 2012

\begin{abstract}
Medications are beneficial for curing or managing acute and chronic illnesses. Medications typically have positive outcomes, although older people are prone to drug-related problems. Community-dwelling seniors are at particularly high risk of polypharmacy, as they tend to receive many prescriptions over time and from different care providers. Continuing-care facility admission presents an excellent opportunity for a comprehensive medication review. A research study was conducted to describe and compare medications taken by community-dwelling seniors prior to and following admission to a continuing-care facility. This pilot project involved data being gathered from the charts of deceased residents, as required by a University Health Research Ethics Board, who had been cared for at one large local continuing-care facility. The facility administrators also approved this study, in part to evaluate their policy to conduct a medication review for all new residents within six weeks of entry. This study revealed a slight but statistically significant reduction in the number of medications following this review. Other issues such as medication interactions and required dosage changes were addressed by this medication review. Although this study was confined to one continuing-care facility and a small number of residents, the findings suggest medication reviews would be beneficial upon admission to all continuing-care facilities, and annually perhaps through other means for older persons living in the community.
\end{abstract}

Keywords: Polypharmacy; Drug Interactions; Older People; Aging; Nursing Homes; Continuing Care; Comparative Research

\section{INTRODUCTION}

Continuing-care facilities are well known as places that provide supportive care for disabled persons, many of whom are elderly [1]. Most continuing-care residents have complex health problems from multiple co-morbidities, often presenting with advanced cognitive and functional impairments. Drug therapy has become an increasingly important modality for curing or managing acute and chronic health problems, but co-morbidities often result in situations where people take multiple medications concurrently. In the case of elderly persons, polypharmacy poses major challenges to optimal drug therapy because of age-related pharmacokinetics and pharmacodynamics, changes that occur when more than one drug is taken daily. The use of multiple medications for the management of multiple co-existing chronic health conditions is now understood as a major factor for prescription-related illness, with McGavock [2] calling prescription-related illness a "scandalous pandemic" instigated by unnecessary prescribing, inappropriate diagnosis, lack of education in pharmacology and therapeutics, and the rapid development and promotion of new drugs. Frazier's [3] literature review revealed an increased prevalence of polypharmacy among older persons; with this identified as a result of many co-existing chronic illnesses, multiple prescribing providers over time and in multiple care settings, the use of more than one pharmacy to obtain medications through, and the shift of previously prescribed drugs to over-the-counter availability.

Multiple medications can pose major problems for people of all ages, but particularly older people. Frazier [3] reported that $70 \%-80 \%$ of older individuals who take more than one drug experience side effects due to their decreased ability to metabolize and excrete multiple medications. Reduced kidney and liver function in old age increases the potential for toxic effects [4]. Older people who are community-dwelling are considered to have the highest risk of polypharmacy and thus prescription-related illnesses [5]. For the small proportion of older people who are admitted to a nursing home or an- 
other type of continuing-care facility, their admission process may involve a comprehensive medication review. Few studies on the effectiveness of these admission medication reviews in continuing-care facilities have been conducted [6]. No studies appear to have been specifically designed to compare the number and types of medications taken by community-dwelling older people before and after their nursing home entry. However, several studies from various countries have demonstrated that medication reviews in continuing care facilities are effective for identifying drug-related problems [7-9]. A descriptive-comparative study of medications taken by older persons prior to and following admission to a large continuing-care facility in Western Canada was undertaken to address this knowledge gap.

\section{LITERATURE REVIEW}

Clearly, polypharmacy is a major concern in many countries, including Canada [3]. This concern explains some recent research, policy, and program developments. In 2009, the Alberta government initiated a call for research aimed at reducing the incidence and impact of polypharmacy. This is the same year that specially trained pharmacists in this Western Canadian province were given the right to review and prescribe medications in certain situations, with polypharmacy thought to be a major factor leading to this professional scope enhancement. Similarly, in 2009, the Alberta government announced a future reduction in their co-share or funding support for the prescribed medications taken by citizens holding supplementary Alberta Blue Cross insurance. This insurance is provided to all residences of Alberta upon request and when monthly insurance premiums are paid; with this insurance provided by a voluntary not-for-profit group that is similar to the Blue Cross programs in other Canadian provinces and territories [10]. The rationale behind this planned reduction was to limit the number of medications taken by older persons, the main group relying on this private insurance program for drug therapy financial assistance.

Interestingly, the existing literature reveals some debate exists about what constitutes polypharmacy. Polypharmacy is described by Salam, Mandal, Kumar, and Almula [11] as the use of multiple medications, some of which are not clinically indicated. More often, polypharmacy is defined by the number of medications taken daily; however, Zurakowski [12] suggested that the practice of trying to stay below a certain number of medications each day can lead prescribing physicians and others into a false sense of security. Zurakowski [12] recommended instead that it is safer for every prescriber to remain alert for any drug-related problems regardless of the total number of medications that a person is taking. Similarly, Ballentine [13] suggested that reducing the number of medications and thus the incidence of polypharmacy should not be the objective, but instead prescribers should practice "rational" polypharmacy rather than "indiscriminate" polypharmacy. Rational or obligatory polypharmacy is considered a form of appropriate prescribing; one where all medications are reviewed for continued indication and positive therapeutic effects, and with the objective of maximizing benefits and minimizing harms to the elderly.

Regardless of this debate, polypharmacy is clearly understood as a serious issue for all people, but particularly older persons. As indicated above, older people typically have multiple co-existing chronic illnesses and age-related organ pathologies, with multiple medications thus indicated. Drug interactions between medications are common, leading to unexpected and at times highly problematic effects. Evidence suggests that drugs from specific classes including antidepressants, neuroleptic agents, benzodiazepines, anticonvulsants, and class IA antiarrhymic medications are consistently associated with falls in older adults [14]. Dizziness, falls, cognitive decline or depression, and weight loss are often secondary to drug side effects [13]. Self-medication often contributes to polypharmacy, as there has been a significant rise in the use of over-the-counter medications, including ones that were previously dispensed only by prescription [15]. Furthermore, complementary or alternative therapies are increasing in popularity. As people generally do not consider herbal and homeopathic remedies to be medications, they often fail to disclose their use to their healthcare providers [15]. Perhaps the greatest problem is that community-dwelling persons, including seniors, typically need to visit multiple physicians, pharmacists, and nurse practitioners for their multiple health problems [13]. As ill people today tend to expect to receive a prescription for their illness (even citing a specific drug by brand name as a result of direct marketing from pharmaceutical companies), many prescribers find it easier to write a prescription than take the time and trouble of educating patients about alternatives to medications such as diet changes, or to educate about the risks of their taking multiple medications [16]. As such, communitydwelling older people are a prime target for evidencebased action on polypharmacy.

\section{RESEARCH METHODOLOGY}

As indicated above, a study was conducted to compare the number and types of medications taken immediately prior to and following admission of community-dwelling seniors to a continuing-care facility. A large public facility (300 beds) was chosen for this study, in part because it has been in operation for over 20 years in a large city in Western Canada, and it has well-established programs and services. One such program is a medication review 
that is expected to be completed within six weeks of entry for all persons admitted. After research ethics and administrative approvals were obtained, data were collected from the charts of deceased older (age 65+) residents of this facility who met the criteria for this studyspecifically that they had been admitted directly from the community and not from a hospital or another nursing home.

The original intention of this study was to review the charts of all live and recently deceased residents (i.e. those who died within one year of the review date), so as to assess the effects of the medication review at this facility. The Health Research Ethics Board (B) prohibited the review of charts of live residents and only permitted one of the two researchers to access the charts as this researcher had a joint-appointment involving that facility. Although these measures were aimed at addressing the privacy and confidentiality needs of vulnerable older adults living in nursing homes, the requirement that only deceased resident charts could be accessed for research purposes significantly reduced the number of charts that could be reviewed.

A total of 225 charts were provided for review, although only 11 satisfied all inclusion criteria. Specifically, the death of the resident had to have occurred less than one year previously, the resident had to have been 65 years of age or older at the time of admission to the nursing home, and the resident was admitted from the community and not a hospital or another nursing home. Data were collected from the 11 charts to answer four research questions:

1) Did the total number of medications taken daily change following nursing home admission medication review?

2) Did any prescribed medications change following nursing home admission medication review?

3) Did the number of potential drug interactions change following the nursing home admission medication review?

4) Did any medication dosages change following nursing home admission medication review?

Data were collected using a simple data collection form which was a computer spreadsheet for rapid data entry. The data were summarized and analyzed using SPSS, a common statistical analysis software program. Descriptive statistics, t-tests, and Pearson correlation analyses were used to answer three of the research questions (1, 2, and 4). An analysis of the drug interactions present prior to and following the medication review was then conducted to answer the third research question, with the Lexi-Comp Online ${ }^{\mathrm{TM}}$ Comprehensive Interaction Analysis Program used to identify and categorize drug interactions. Finally, to determine pre/post changes to the dosage of medications, and to determine any changes in the types or classifications of medications prescribed, a manual comparison of data obtained from each chart was performed.

\section{RESULTS}

\subsection{Descriptive Findings}

As indicated, 225 charts were made available for review. It had been anticipated that data would be gathered from 200 to 250 charts, a number similar to the number of deaths and thus number of admissions each year to this facility. This target number was also based on the expectation that at least one half of all admissions to nursing homes are from the community. However, only $4.9 \%$ of these charts met the criteria for review $(n=11)$.

Of the 11 residents, $36.4 \%(n=4)$ were male and $63.6 \%$ female $(n=7)$. They ranged in age from 65 to 93 years old, with a mean age of 82.0. Their most common primary diagnoses were varied: Osteoarthritis, depression, neuropathy, hypertension, chronic obstructive pulmonary disease, coronary artery disease, Parkinson's disease, dementia, renal failure, and anemia. Co-morbidities were present among all residents, with 6.6 the average number of co-morbidities. For each resident, their medication review was completed 32 to 142 days after admission, with a mean of 59 days.

\subsection{Medication Number}

The average number of all regular and periodic or PRN medications taken daily at the time of admission was 11.1 and 10.9 following the medication review, a small but statistically significant decrease $(\mathrm{t}=5.565$, $\mathrm{df}=10$, $\mathrm{p}$ $=0.000$ ). Significant results were also obtained when a t-test was used for a pre/post comparison of only those medications taken regularly each day $(\mathrm{t}=4.878$, $\mathrm{df}=10$, $\mathrm{p}=0.001)$.

Pearson correlation analyses were then undertaken to assess for relationships between resident age and number of medications taken both before and after the medication review. The first did not find a relationship between age and total number of medications (regular and periodic medications) on admission $(\mathrm{R}=-0.414, \mathrm{p}=0.205)$. The second did not find a relationship between age and the number of regular medications on admission $(\mathrm{R}=$ $-0.505, \mathrm{p}=0.113$ ). The third also did not find a relationship between age and the total number of medications (regular and PRN medications) after the medication review $(\mathrm{R}=-0.333, \mathrm{p}=0.318)$. The final correlation similarly did not find a relationship between age and the number of regular daily medications taken after the medication review $(\mathrm{R}=-0.500, \mathrm{p}=118)$.

A second series of Pearson correlation analyses were also undertaken to assess for relationships between the number of co-morbidities and number of medications. 
These four correlations were also non-significant. The first test involved co-morbidities and the total number of medications (regular and periodic medications) taken on admission $(\mathrm{R}=0.080, \mathrm{p}=0.816)$. The second involved co-morbidities and the number of regular daily medications taken on admission $(\mathrm{R}=0.271, \mathrm{p}=0.421)$. The third correlation involved co-morbidities and the total number of medications (regular and periodic medications) taken after the medication review $(\mathrm{R}=0.151, \mathrm{p}=0.658)$. The final correlation involved co-morbidities and the number of regular daily medications taken after the medication review $(\mathrm{R}=0.336, \mathrm{p}=0.312)$.

\subsection{Types of Medications and Dosage Changes}

Using the list of medications recorded, classifications were assigned to each medication taken before and after the medication review, with these classifications compared on a case-by-case basis. Few changes in each resident's pre/post classifications or general types of medications were found. Generally, the type of medication taken remained the same after the medication review. The most common drugs prescribed were in the alimentary, cardiovascular, and nervous system classifications. Aspirin and acetaminophen were the most common overthe-counter medications. However, a considerable number of dosage changes were found as a result of the medication review, with just over $50 \%$ of medications having a dosage change, most of which were minor however.

\subsection{Number of Drug Interactions}

Using the Lexi-Comp Online ${ }^{\mathrm{TM}}$ Comprehensive Interaction Analysis Program, potential drug interactions both prior to and following the medication review were found to be common, with 72 in total. Most often, these drug interactions had a risk rating of "C", indicating that the resident's clinical state and their drug therapy should be monitored, as the drugs could possibly interact with each other in a clinical significant manner, but that the benefits of therapy would normally prevail over the potential risks. This "C" risk rating also indicates that a careful monitoring plan should be implemented to ensure that negative effects of therapy would be rapidly recognized and then lead to dosage or medication adjustments. Prior to the medication review, $76 \%$ of the interactions identified had a risk rating of "C", compared to $78 \%$ following the medication review. The next most common risk ratings were "B" (14\%), a rating indicating no action required, and " $\mathrm{D}$ " (10\%), a rating indicating therapy modification should be considered. Among the residents who had a reduction in the number of medications following their medication review, a decline in the total number of drug interactions was noted, with all D-rated interactions eliminated.

\section{DISCUSSION}

This study revealed a small, but statistically significant, reduction in the number of medications taken by older people following their nursing home admission medication review. This finding suggests medication reviews conducted following admission to continuing-care facilities are an effective intervention for reducing polypharmacy and its risks. A larger study of continuing-care residents by Khunti and Kinsella [9] similarly found a single comprehensive medication review leads to a significant reduction in the number of medications, and therefore cost savings.

Although this study found dosage changes were common and some drug interactions were eliminated or noted for continuing assessment, one finding of concern was that the conference for the medication review took place an average of 59 days after admission. This time frame is outside the expected six weeks, but it is possible that this longer timeframe is more appropriate to thoroughly assess the clinical relevance of each medication and the resident's overall state of health-particularly as relocating to a nursing home is a major move, with many potential negative and positive impacts [17]. It is also possible that practical issues, such as an inability to gather the large interdisciplinary team together for this case conference led to this delay. In this specific facility, the case conference involves multiple members of the healthcare team, with this case conference not only used to complete the medication review but also to reassess the initial care plan for the resident. Care plans are typically drafted at admission through discussions with the resident and family (whenever possible) and through relying on previous care plans (if they are provided). As such, this case conference is a very important event that cannot and perhaps should not be rushed.

The present study found few pre-post changes in the types of medications taken by the residents, with this indicating that the medications taken by these older chronically-ill people were not inappropriately prescribed, a major finding as it indicates that polypharmacy may not be as much of a concern as is widely assumed. Minor adjustments in dosage were more common, with this also indicating another positive outcome of the admission medication review. Dosage changes could be the most important focus of drug reviews, as the same dose of a medication may be taken for many years despite weight loss with aging or illness [18]. It is also important to note that medications with alimentary, cardiovascular, and nervous system classifications were the most commonly prescribed. These drug findings are consistent with the results of a larger study by Randall and Bruno [19]. Ran- 
dall and Bruno also concluded that these medication classes are the most problematic for adverse drug reactions. As such, health care providers should be attentive to drug-related illnesses among older persons with the co-morbidities of cardiovascular disease, psychiatric conditions, and gastro-intestinal disorders.

As indicated, acetaminophen and aspirin were found in the present study to be the most common over-thecounter medications prescribed and thus taken by residents. A study by Kaufman, Kelly, Rosenburg, Anderson, and Mitchell [20] similarly revealed acetaminophen and aspirin were common medications used by older people, as well as ibuprofen. They warned that these common over-the-counter medications contribute to polypharmacy, as they often interact with or duplicate the effects of drugs prescribed for cardiovascular conditions. More care providers should know of this concern, as these common over-the-counter medications are generally viewed as harmless.

This chart review study also revealed that nursing home entry most often followed a transfer from an acutecare hospital or another continuing-care facility. Several other studies have found relocations from one facility to another are common for older adults, including those with acute or chronic health conditions [21,22]. Important questions about the nature of these relocations and the impact of these relocations, such as the possibility of reduced care continuity and medication errors, have already been raised. An investigation conducted by Boockvar et al. [23] found medication alterations are common during care setting transitions and also after any relocations, with these resulting in adverse drug interactions and other drug problems. Adverse events due to medication changes or lack of communication were found in $20 \%$ of cases involving a transfer between acute-care and continuing-care facilities [23]. Boockvar et al. [23] suggested that this issue may be due to inaccurate or incomplete communication between facilities about medication regimens, with facilitates not generally affiliated and thus not sharing medical records or even drug forms. Simple written lists of medications and other medication-related information could prevent this issue.

Unfortunately, the present study is severely limited by the small sample, a concern that raises considerable doubt about the findings. Studies involving a larger sample or an entire population may or may not reveal a significant reduction in medications after nursing home admission. Another limitation of this study is that it was only conducted at one care facility, a factor considerably reducing the generalizability of findings. The small sample also limited the possibility of identifying clear associations between the number of medications taken pre or post admission with factors such as age and co-morbidities. Given the widespread and rising concerns about poly- pharmacy, there is much need to replicate this pilot study to ensure a larger sample, such as by reviewing charts at multiple continuing-care facilities or by carrying out this study in jurisdictions that permit chart reviews of live nursing home residents. Methodology refinements could also be made with regard to which medications are studied, as Finkers, Maring, Boersma, and Taxis' [6] study indicates vaginal, eye, ear, and topical drugs are unlikely to contribute to drug-related interactions.

Future research should also clearly establish whether medication reviews are commonly conducted after admission to a nursing home or other types of continuing-care and rehabilitation or sub-acute care facilities. Other studies could focus on medication reviews undertaken in community care settings, such as at primary care or nurse practitioner clinics. In addition, future research needs to establish the effectiveness of all such reviews for identifying and addressing polypharmacy. Studies could also be done on organizational and/or human resource factors that influence prescribing practices and medication regimens for older people. It would also be helpful to explore the intended aim or aims of medication reviews, and to learn why these reviews were initiated in the first place.

All of this research is needed, as continuing-care residents typically suffer from advanced physical and/or cognitive disabilities, with advocates needed to ensure an appropriate clinical reason for each medication and to ensure that cost-cutting does not deprive these persons of needed therapeutic drugs. Further studies could also determine which health professionals are involved in medication reviews, and if nurses or others are effective polypharmacy prevention advocates for continuing-care residents. Dunn [24] suggested that nurses should independently conduct medication reviews and should also advocate for routine medication reviews, such as those on admission to nursing homes. Nurses and others can also create or advocate for the development of patient education resources to encourage self-review of medications, so that individuals can quickly and easily identify interactions, contraindications, and side effects. Community health nurses have the additional opportunity to collaborate with community pharmacists and family physicians or nurse practitioners to ensure timely medication reviews are undertaken for community-dwelling older persons. Home care nurses are in another ideal position to address the drug information needs of older persons and their families, and to conduct medication reviews to ensure that seniors receive medications in the correct dose and form to achieve favorable pharmacotherapy outcomes [25].

Other studies need to be done to provide evidence to support the formal integration of pharmacists into the healthcare team to minimize the harmful effects of poly- 
pharmacy through pharmacist-led medication reviews such as annually or whenever a new medication is prescribed [26]. Dilks [27] emphasized the emerging role of pharmacists either working independently or in multidisciplinary teams for medication management of persons with multiple chronic illnesses. Dilks' study of 468 patient encounters, involving community-dwelling individuals identified as being at pharmaceutical risk, found that pharmacists made 158 interventions which resulted in improved conformity with the prescribed drug plan, 73 interventions where unnecessary medications were stopped, and 65 interventions where the pharmacist suggested a new or different medication in consultation with the family physician. Dilks concluded that the role of the pharmacist is vital. Their knowledge and training allows them to provide a comprehensive review of medications, including identifying inappropriate use and dosing systems and drug interactions.

Regardless of who does what, drug-related illnesses are important to detect and extremely important to prevent. Given the commonality of co-morbidities and thus the risk of polypharmacy, reliable and validated assessment tools are needed for this to occur. The explicit criteria developed by Beers, Ouslander, Rollingher, Reubenen, Brooks, and Beck [28] for determining inappropriate medication use among older people is therefore of considerable interest. This was a major development for reviewing medications, such as by determining which medications should be avoided, and for identifying safe doses and frequencies that should not be exceeded. Their 1997 update was also aimed at enhancing the applicability of this tool for ambulatory older persons. The 1997 criteria details 28 inappropriate medications and 35 inappropriate medications for 15 common health conditions [29]. Their criteria was again updated in 2003 to make the criteria applicable for both ambulatory and continuing-care settings [30].

In 1997, a Canadian panel also developed similar criteria for identifying inappropriate medications [31]. They pointed out 18 inappropriate medications regardless of the individual's diagnosis, 16 inappropriate drug-disease interactions, and 6 inappropriate drug-drug interactions. Several other assessment tools, including the "brown bag approach", the use of mnemonics such as SAIL or TIDE, and the 10-step approach have been developed to assess for polypharmacy [16,32,33]. Nurses and other healthcare professionals should use these objective tools and systematic medication reviews to improve patient outcomes by influencing prescribing practices that reduce indiscriminant polypharmacy and drug-related illnesses.

\section{CONCLUSION}

This small descriptive-comparative pilot study, comparing the number and types of medications taken by resi- dents prior to and following admission from the community to a continuing-care facility, revealed some findings of relevance for future research and consideration. A significant reduction in medications was found, indicating that polypharmacy can be addressed by a comprehensive medication review conducted soon after admission. Continuing-care facilities thus have a significant opportunity to reduce polypharmacy and drug-related illness. The problem of polypharmacy and drug-related illness will require more than just a medication review after admission to nursing homes, however. Statistics Canada [34] estimates that there are 4.8 million Canadians who are 65 years of age or older, a number that is expected to double by 2036 to reach 10.4 million seniors. By 2051, one in four Canadians is expected to be 65 years of age or older. Population aging is also occurring globally, with the proportion of older persons rising from $8 \%$ in 1950 to $10 \%$ in 2000, and projected to reach $21 \%$ in 2050 [35]. With an aging global population, it is prudent to adopt strategies now that focus on illness prevention, health promotion, and maintenance of health. Proactive or upstream approaches that reduce the incidence of acute and chronic illnesses, and routinely prevent polypharmacy issues will ultimately prove more beneficial than waiting until the person is so ill that they need to move into a nursing home and then have a medication review conducted.

\section{ACKNOWLEDGEMENTS}

Research funding for this study from the Caritas/Covenant Research Trust Fund is gratefully acknowledged. The assistance of facility staff and administrators in facilitating this study is also gratefully acknowledged.

\section{REFERENCES}

[1] Wilson, D. and Truman, C. (2004) Long-term-care residents. Concerns identified by population and care trends. Canadian Journal of Public Health, 95, 382-386.

[2] McGavock, H. (2004) Prescription related illness-A scandalous pandemic. Journal of Evaluation in Clinical Practice, 10, 491-497.

doi:10.1111/j.1365-2753.2003.00471.x

[3] Frazier, S.C. (2005) Health outcomes and polypharmacy in elderly individuals. Journal of Gerontological Nursing, 31, 4-11.

[4] Papaioannou, A., Clarke, J., Campbell, G. and Bedard, M. (2000) Assessment of adherence to renal dosing guidelines in long term care facilities. Journal of the American Geriatrics Society, 48, 1470-1473.

[5] Hastings, S.N. and White, H.K. (2005) Medication review in the nursing home: Can stopping one drug make all the difference? Annals of Long Term Care, 13, 33-35.

[6] Finkers, F., Maring, J.G., Boersma, F. and Taxis, K. 
(2007) A study of medication reviews to identify drugrelated problems of polypharmacy patients in the Dutch nursing home setting. Journal of Clinical Pharmacy and Therapeutics, 32, 469-476.

doi:10.1111/j.1365-2710.2007.00849.x

[7] Furniss, L., Burns, A., Craig, S.K., Scobie, S., Cooke, J. and Faragher, B. (2000) Effects of pharmacist's medication review in nursing homes: Randomized controlled trial. British Journal of Psychiatry, 176, 563-567. doi:10.1192/bjp.176.6.563

[8] Halvorsen, K.H., Ruths, S., Granas, A.G. and Viktil, K.K. (2010) Multidisciplinary intervention to identify and resolve drug-related problems in Nowegian nursing homes. Scandinavian Journal of Primary Health, 28, 82-88. doi:10.3109/02813431003765455

[9] Khunti, K. and Kinsella, B. (2000) Effect of systematic review of medication by general practitioner on drug consumption among nursing-home residents. Age and Ageing, 29, 451-453. doi:10.1093/ageing/29.5.451

[10] Blue Cross Canada (no date) Enjoy the benefits of good health. http://www.bluecross.ca/

[11] Salam, A., Mandal, S., Kumar, A. and Almula, A.A. (2008) Polypharmacy: Cure or curse? Quality in Ageing, 9, 24-28. doi:10.1108/14717794200800004

[12] Zurakowski, T. (2009) The practicalities and pitfalls of polypharmacy. The Nurse Practitioner, 34, 36-41. doi:10.1097/01.NPR.0000348320.38365.59

[13] Ballentine, N.H. (2008) Polypharmacy in the elderly. Critical Care Nursing, 31, 40-45.

[14] Tinetti, M.E. (2003) Clinical practice. Preventing falls in elderly persons. New England Journal of Medcine, 348, 42-49. doi:10.1056/NEJMcp020719

[15] Fulton, M.M. and Allen, E.R. (2005) Polypharmacy in the elderly: A literature review. Journal of the American Academy of Nurse Practitioners, 17, 123-132. doi:10.1111/j.1041-2972.2005.0020.x

[16] Prybys, K., Melville, K., Hanna, J., Gee, A. and Chyka, P. (2002) Polypharmacy in the elderly: Clinical challenges in emergency practice: Part 1 overview, etiology and drug interactions. Emergency Medicine Reports, 23, 145-153.

[17] Hodgson, N., Freedman, V.A. and Granger, D.A. (2004) Relocation puts elderly nursing home residents at risk of stress, although the stress is short lived. Journal of the American Geriatrics Society, 52, 1856-1862. doi:10.1111/j.1532-5415.2004.52505.x

[18] Huffman, G.B. (2002) Evaluating and treating unintentional weight loss in the elderly. American Family Physician, 65, 640-654.

[19] Randall, R.L. and Bruno, S.M. (2006) Can polypharmacy reduction efforts in an ambulatory setting be successful? Clinical Geriatrics, 14, 33-35.

[20] Kaufman, D., Kelly, J., Rosenberg, L., Anderson, T.E. and Mitchell, A.A. (2002) Recent patterns of medication use in the ambulatory adult population in the United States: The Slone survey. Journal of the American Medical Association, 287, 337-344. doi:10.1001/jama.287.3.337

[21] Coleman, E.A. (2003) Falling through the cracks: Challenges and opportunities for improving transitional care for persons with continuous complex care needs. Journal of the American Geriatrics Society, 51, 549-555. doi:10.1046/j.1532-5415.2003.51185.X

[22] Murtaugh, C.M. (2002) Transitions through post-acute and long-term care settings: Patterns of use and outcomes for a national cohort of elders. Medical Care, 40, 227-236. doi:10.1097/00005650-200203000-00006

[23] Boockvar, K., Fishman, E., Kyriacou, C.K., Monias, A., Gavi, S. and Cortes, T. (2004) Adverse events due to discontinuation in drug use and dose changes in patients transferred between acute and long term care facilities. Archives of Internal Medicine, 164, 545-550. doi:10.1001/archinte.164.5.545

[24] Dunn, C.M. (2002) Assessing and preventing medication interactions. Home Healthcare Nurse, 20, 105-111. doi:10.1097/00004045-200202000-00008

[25] Cowan, D., While, A., Roberts, J. and Fitzpatrick, J. (2002) Medicines management in care homes for older people: The nurse's role. British Journal of Community Nursing, 7, 634-638.

[26] Stuijt, C.M., Franssen, E.J.F., Egberts, A.C.G. and Hudson, A.H. (2008) Appropriateness of prescribing among elderly patients in a Dutch residential home. Drugs and Aging, 25, 947-954. doi:10.2165/0002512-200825110-00005

[27] Dilks, S. (2007) The emerging role of the domiciliary pharmacist in Devon. Journal of Integrated Care, 15, 20-25. doi:10.1108/14769018200700035

[28] Beers, M., Ouslander, J., Rollingher, I., Reuben, D., Brooks, J. and Beck, J. (1991) Explicit criteria for determining inappropriate medication use in nursing home residents. Archives of Internal Medicine, 151, 1825-1832. doi:10.1001/archinte.1991.00400090107019

[29] Beers, M. (1997) Explicit criteria for determining potentially inappropriate medication use by the elderly: An update. Archives of Internal Medicine, 157, 1531-1537. doi:10.1001/archinte.1997.00440350031003

[30] Fick, D.M., Cooper, J.W., Wade, W.E., Waller, J.L., Maclean, R. and Beers, M.H. (2003) Updating he Beers criteria for potentially inappropriate medication use in older adults. Archives of Internal Medicine, 163, 27162724. doi:10.1001/archinte.163.22.2716

[31] McLeod, P.J., Huang, A.R., Tamblyn, R.M. and Gayton, D.C. (1997) Defining inappropriate practices in prescribing for elderly people: A national consensus panel. $\mathrm{Ca}$ nadian Medical Association Journal, 156, 385-391.

[32] Werder, S.F. and Preskorn, S.H. (2003) Managing polypharmacy: Walking the fine line between help and harm. Journal of Family Practice, 2, 24-36.

[33] Carlson, J. E. (1996) Perils of polypharmacy: 10 steps to prudent prescribing. Geriatrics, 51, 26-32.

[34] Statistics Canada (2010) Population estimates and projections. http://www40.statcan.ca/101/ind01/13_3867_3433-eng.ht m?hili demo23

[35] United Nations (2007) World population ageing 2007. http://www.un.org/esa/population/publications/WPA2007 /wpp2007.htm 